\title{
PERSISTENCE OF FORM AND THE VALUE GROUP OF REDUCIBLE CUBICS
}

\author{
P. D. T. A. ELLIOTT
}

\begin{abstract}
It is proved that the values of $x\left(x^{2}+c\right), c \neq 0$, at positive integers, multiplicatively generate the positive rationals. Analogs in rational function fields are obtained.
\end{abstract}

1. Let $Q^{*}$ be the multiplicative group of positive rational numbers. Let $r_{1}$, $r_{2}, \ldots$, be a sequence of positive rationals, and $\Gamma$ the subgroup of $Q^{*}$ which they generate. Let $G$ be the quotient group $Q^{*} / \Gamma$. This group $G$ reflects the extent to which an arbitrary positive integer has a multiplicative representation by the $r_{n}$. Since $Q^{*}$ is freely generated by the positive prime numbers, $G$ models an arbitrary denumerable abelian group, and an algorithm to determine its structure cannot be given. However, this situation could change if the $r_{n}$ were given enough algebraic properties.

Let $F(x)$ be a rational function $P_{1} / P_{2}$, the $P_{i}$ in $Z[x]$ and having positive leading coefficients. Let $\theta$ be a nonnegative real number, and choose for the sequence of rationals $r_{n}$ the positive values among the $F(t)$ as $t$ runs through the integers greater than $\theta$. In my book $[\mathbf{4}]$ and paper [5] I made early versions of the following conjectures:

(i) For all sufficiently large $\theta, G$ is independent of $\theta$.

(ii) If $F$ is an irreducible polynomial, or more generally a squarefree rational function, then $G$ is the direct sum of a free group and a finite group.

A consequence of these conjectures would be that those positive integers $m$ which have representations of the form

$$
m^{k}=\prod_{i=1}^{d} F\left(t_{i}\right)^{\varepsilon_{i}}, \quad \varepsilon_{i}= \pm 1,
$$

with positive integers $t_{i}$, would possess infinitely many of them. Moreover the same fixed value of $k$ could be taken for all $m$. To some extent this is a multiplicative analogue of Waring's problem (cf. Vaughan [8]).

In an abuse of notation I shall write $Q^{*} / \Gamma(F(n))$ for $G$, notationally suppressing the possible dependence on $\theta$.

I have verified these conjectures for the following classes of functions.

A. $F(x)=x^{2}+b x+c$ for integers $b, c$ with $b^{2} \neq 4 c$. Thus when $F(x)=x^{2}+1$, the group $G$ is free with generators the $p(\bmod \Gamma)$ for primes $p \equiv 3(\bmod 4)$.

B. $F(x)=\prod_{j=1}^{k}\left(x-a_{j}\right)^{b_{j}}$ with distinct integers $a_{j}$, and integers $b_{j}$ which have highest common factor 1 . In this case $G$ is trivial.

Received by the editors December 12, 1985.

1980 Mathematics Subject Classification (1985 Revision). Primary 10H99, 10K20, 10 M05.

Partially supported by NSF contract DMS-8500949. 
In addition, I have verified conjecture (ii) when

C. $F(x)=(a x+b) /(c x+d)$ where $a>0, c>0, b, d$ are integers for which $a d \neq b c$. The free group then has finite rank, and the finite group can have arbitrarily large order.

Case B was established in [6]. A second presentation of the argument along with the (considerably complicated) consideration of $\mathrm{C} I$ included in my book [4]. I shall sketch a proof of case $A$ below.

In this paper I consider the cases $F(x)=w(x)=x^{k}\left(b x^{2}+a\right)^{l}$ where $a$ and $k$ are nonzero integers, $b, l$ are positive integers. In particular I establish

THEOREM 1. Both conjectures are valid if $F(x)$ has the form $x^{-1}\left(b x^{2}+a\right)$ or $x\left(x^{2}+a\right)$ for integers $a \neq 0, b>0$.

2. Let $Q(x)^{*}$ be the multiplicative group generated by the rational functions $P_{1} / P_{2}$ in the previous section. For a given rational function $S(x)$ in $Q(x)^{*}$ let $\Delta(S(x))$ be the subgroup of $Q(x)^{*}$ which is generated by the $S(K(x))$ where $K(x)$ is a polynomial in $Z[x]$ with positive leading coefficient. Define the quotient group $H(S(x))=Q(x)^{*} / \Delta(S(x))$.

One might hope to determine the group $Q^{*} / \Gamma(F(n))$ by investigating its polynomial analogue $H(F(x))$, and in this way obtain parametrized product representations. In fact I shall establish

THEOREM 2. The group $H\left(x^{-1}\left(b x^{2}+a\right)\right)$ with $a \neq 0, b>0$ is trivial, but $H\left(x\left(x^{2}+a\right)\right)$ is cyclic of order 3 , generated by the image of $x$.

Following the proofs of these theorems I discuss related results, and give applications to the study of Dirichlet character values.

3. We say that a rational function $F(x)$ has persistence of form if there are distinct polynomials $K_{i}(x)$ in $Z[x]$, with positive leading coefficients, and integers $d_{i}$ not all zero, so that

$$
\prod_{i=1}^{r} F\left(K_{i}(x)\right)^{d_{i}}=\text { constant }
$$

holds identically. It is not clear which rational functions have persistence of form, nor how many such relations can exist for a given function $F(x)$. Some pause is induced by noting that the composition of two irreducible polynomials can be reducible. If $f(x)$ is in $Z[x]$, then Taylor's theorem shows that $f(f(x)+x)$ is divisible by $f(x)$ in $Z[x]$, and an example is furnished by $f(x)=x^{2}+1$.

In this section I consider the persistence of form of quadratic polynomials.

LEMMA 1. Let $h(t)=\alpha t^{2}+\beta t+\gamma, \alpha \neq 0$. Then

$$
h\left(M^{-1} h(t)+t\right)=M^{-2} \alpha h(t) h\left(t+M \alpha^{-1}\right) .
$$

ProOF. This identity, considered to hold between rational functions of $t, \alpha, \beta$, $\gamma$ and $M$, can be verified directly.

As an example in the application of Lemma 1, I establish the conjectures for the irreducible quadratic polynomials $h(x)$ of the form $x^{2}+b x+c, c \neq 0$.

Let $\phi$ denote the canonical map $Q^{*} \rightarrow Q^{*} / \Gamma\left(n^{2}+b n+c\right)$, for some fixed underlying $\theta>|c|$. Let $\bar{r}$ denote the image of a rational number $r$ under this map. 
Let $s$ be the product of the $h(r)$ with integers $r,|r| \leq b+\theta$. Let $q$ be a prime, not dividing $s$, for which the Legendre symbol satisfies $\left(\left(b^{2}-4 c\right) / q\right)=0$ or 1 . The polynomial $x^{2}+b x+c$ splits $(\bmod q)$, say as $(x-u)(x-v)$ where $u, v$ can be represented by integers in the interval $[\theta+|b|+1, q-|b|-1]$. Since $u+v \equiv-b$ $(\bmod q)$ and $0<u+v+b<2 q$, we have $u+v=q-b$. Without loss of generality we shall assume that $0<u<(q+|b|) / 2$.

Under the map $\phi$ we obtain $\bar{q}=\phi\left(q^{-1} h(u)\right)$ where $0<q^{-1} h(u)<q$ for all large enough $q$. The subgroup $G_{1}$ of $G$ which is generated by these $\bar{q}$ is thus finitely generated.

Moreover, applying Lemma 1 with $\alpha=1, M=q, t=u$ we obtain $\bar{q}^{2}=\overline{1}$. Thus $G_{1}$ is finite, of order $k$ say.

Consider now a relation

$$
\prod_{i=1}^{I} \bar{p}_{i}^{\lambda_{i}} \prod_{j=1}^{J} \bar{q}_{j}^{\mu_{j}}=\overline{1}
$$

with integers $\lambda_{i}, \mu_{j}$, primes $p_{i}$ for which $\left(\left(b^{2}-4 c\right) / p_{i}\right)=-1$, and primes $q_{j}$ for which this symbol has value 0 or 1 . Raising everything to the $k$ th power gives

$$
\phi\left(\prod_{i=1}^{I} p_{i}^{\lambda_{i} k}\right)=\overline{1} .
$$

A relation of this kind is possible only if each $p_{i}$ divides $n_{i}^{2}+b n_{i}+c$ for some integer $n_{i}$, and this the condition on the Legendre symbol rules out. Thus every $\lambda_{i}=0$.

It is clear that $G$ is the direct sum of a free group and of $G_{1}$. The finite $G_{1}$ has order which is a power of 2 .

Suppose now that $Q(m)>0$ for all integers $m>z$. Since

$$
Q(m)=Q(Q(m)+m) / Q(m+1)
$$

we see that if the ratio of two rationals has a product representation in terms of $Q\left(n_{i}\right)$ with integers $n_{i} \geq \theta>z$, then it also has such a representation with the stronger requirement $n_{i} \geq \theta+1$. For $\theta>z$ the groups $G$ may thus be identified with each other.

In particular $Q^{*} / \Gamma\left(n^{2}+1\right)$ is free for all $\theta$.

LEMMA 2. Let $Q(x)=b x^{2}+a$ with integers $b>0$ and $a \neq 0$. There are positive integers $D_{0}, D, D_{i}, i=1,2,3$, so that

$$
a^{2} Q\left(D_{0} x Q(D x)\right)=Q\left(D_{1} x\right) Q\left(D_{2} x\right) Q\left(D_{3} x\right) .
$$

PROOF. The strategy behind this lemma is to compose a quadratic polynomial with a cubic polynomial in such a way that the resulting polynomial splits into three quadratics. Consideration of algebraic extensions of the rationals shows that the cubic must be reducible.

Consider $Q\left(M^{-1} x\left(b x^{2}+a\right)+x\right)$ where $M$ may be thought of as a rational number. It has the alternative representation

$$
M^{-2} Q(x)\left(b^{2} x^{4}+b x^{2}[a+2 M]+M^{2}\right) .
$$

The polynomial of degree 4 , considered as a quadratic in $b x^{2}$, is reducible if $(a+2 M)^{2}-4 M^{2}$ is a square. Choosing $M$ to be of the form $a\left(\rho^{2}-1\right) / 4$ for a rational $\rho$ will ensure that this condition is satsified, and the polynomial splits as

$$
\left[b x^{2}+a(\rho+1)^{2} / 4\right]\left[b x^{2}+a(\rho-1)^{2} / 4\right] .
$$


With this choice of $M, M+a=a\left(\rho^{2}+3\right) / 4$. Here $\rho^{2}+3=y^{2}$ has only $\rho= \pm 1$, $y= \pm 2$ as integral solutions, but has infinitely many rational solutions given by $\rho=\frac{1}{2}(3 t-1 / t), y=\frac{1}{2}(3 t+1 / t)$. It is convenient to note that $\rho>0$ if $t>1 / \sqrt{3}$; and that since $2 t(\rho-1)=(3 t+1)(t-1)$ for positive rational values of $t, \rho>1$ if and only if $t>1$.

Noting further that

$$
b x^{2}+a\left(\frac{\rho-1}{2}\right)^{2}=\left(\frac{\rho-1}{2}\right)^{2} Q\left(\frac{2 x \operatorname{Sign} a}{\rho-1}\right)
$$

where

$$
\text { Sign } a= \begin{cases}1 & \text { if } a>0 \\ -1 & \text { if } a<0\end{cases}
$$

we obtain the identity

$$
a^{2} Q\left(\frac{x(3 t+1 / t)^{2}}{4 M} Q\left(\frac{2 x}{3 t+1 / t}\right)\right)=Q(x) Q\left(\frac{2 x}{\rho+1}\right) Q\left(\frac{2 x \operatorname{Sign} a}{\rho-1}\right) .
$$

We set

$$
x=(a / 4)\left(\rho^{2}-1\right)(3 t+1 / t)(2 t)^{r} z
$$

and fix $r$ at a value sufficiently large that all the polynomials belong to $Z[t, z]$. If $a>0$, we choose $t$ to be a rational number exceeding 1 , if $a<0$, we choose $t$ to be a rational number in the interval $1 / \sqrt{3}<t<1$. This ensures that both $\rho$ and $a(\rho-1)$ are positive. Replacing $z$ by $D_{4} u$ for a suitably chosen positive integer $D_{4}$ we obtain the desired identity with $u$ in place of $x$.

It is interesting that the constants $D$ and $D_{i}, i=1,2$, are constant multiples of $a$, while $D_{3}$ is a constant multiple of $|a|$. $D_{0}$ is an absolute constant. They satisfy $|a| D_{0} D^{2}=D_{1} D_{2} D_{3}$. [5].

Other examples in the persistence of form of quadratic polynomials are given in

4. Proof of Theorems 1 and 2 for $x^{-1}\left(b x^{2}+a\right)$.

LEMMA 3. Let $g=k(k+2 l)(k, l)^{-1}$. There are polynomials $K_{i}$ in $Z[x]$ with positive leading coefficients and of degree at most 3 , so that

$$
x^{g}=\prod_{i=1}^{c} w\left(K_{i}\right)^{\varepsilon_{i}}, \quad \varepsilon_{i}= \pm 1 .
$$

PROOF. Raising the identity of Lemma 2 to the $l$ th power gives

$$
x^{-3 k} \prod_{i=1}^{3} D_{i}^{-k} w\left(D_{i} x\right)=a^{2 l}\left(D_{0} x Q(D x)\right)^{-k} w\left(D_{0} x Q(D x)\right) .
$$

In turn we take $l(k, l)^{-1}$ powers in this equation and obtain a representation

$$
x^{g} L=\prod_{i=1}^{h} w\left(J_{i}(x)\right)^{\varepsilon_{i}}
$$

with a positive constant $L$ and polynomials $J_{i}(x)$ in $Z[x]$, of degree at most 3 , with positive leading coefficients. 
Employing this identity twice, once with $x=1$, gives the desired identity of Lemma 3.

In the application of Lemma 3 it is sometimes convenient to be able to assert that all integer specializations of the polynomials $K_{i}$ exceed a given constant. This may be obtained by replacing $x$ in the above identity involving the $J_{i}(x)$ with $E x$ for a suitable integer $E$, or by also employing the identity with $x$ everywhere replaced by $x^{2}$.

In the case $-k=1=l$ we have $g=1$, giving the triviality of $H\left(x^{-1}\left(b x^{2}+a\right)\right)$, and so that of $Q^{*} / \Gamma\left(n^{-1}\left(b n^{2}+a\right)\right)$ for all $\theta$. For the cubics $x\left(x^{2}+a\right)$ we obtain $g=3$.

5. In this and all following sections the relation $g_{1} \sim g_{2}$ between two members of a group $G$ means that their ratio $g_{1} g_{2}^{-1}$ belongs to the kernel of a given group homomorphism $G \rightarrow H_{1}$, or of a composition of homomorphisms $G \rightarrow H_{1} \rightarrow H_{2} \rightarrow$ $\cdots$.

We shall apply, many times, the identity

$$
B(B(x)+x)=B(x) B(x+1)
$$

which is valid for all quadratic polynomials $B(x)$ in $Z[x]$ which are of the form $x^{2}+\beta x+\gamma$. It is the case $\alpha=1=M$ of Lemma 1 .

In this section and until further notice $b=1$ so that $w(x)=x^{k}\left(x^{2}+a\right)^{l}$.

LEMMA 4. Under the map $Q\left(x^{*}\right) \rightarrow H(w(x))$ we have $\left(x^{2}+a-1\right)^{k l s} \sim$ $\left(x^{2}-1\right)^{k l s}$ with $s=(k, l)^{-1}$.

PROOF. Beginning with the relation $\left(x^{2}+a\right)^{l} \sim x^{-k}$ we apply identity (1) with $B(x)=x^{2}+a$ to obtain

$$
\left(x^{2}+x+a\right)^{-k} \sim\left(B\left(x^{2}+x+a\right)\right)^{l} \sim(x(x+1))^{-k} .
$$

Using the left and right ends of this expression and applying identity (1), this time with $B(x)=x^{2}+x+a$, yield

$$
\left((x+1)^{2}+a-1\right)^{k}\left((x+1)^{2}+a\right)^{k} \sim\left(x(x+1)^{2}(x+2)\right)^{k} .
$$

We replace $x$ by $x-1$ and eliminate between the resulting expression and the first relation to obtain

$$
\left(x^{2}+a-1\right)^{k l s} \sim\left(x^{2}-1\right)^{k l s} x^{k s(k+2 l)} .
$$

An application of Lemma 3 now gives the desired result.

LEMMA 5. Let $\beta=k l(k, l)^{-1}$. Under the composition of maps $Q(x)^{*} \rightarrow$ $H(w(x)) \rightarrow \beta H(w(x))$ we have

$$
\begin{gathered}
x^{2}+x+a-m^{2}+m \sim(x-m+1)(x+m), \\
x^{2}+a-m^{2} \sim(x-m)(x+m)
\end{gathered}
$$

for $m=1,2, \ldots$.

The second homomorphism in this sequence raises elements to their $\beta$ th power.

PROOF. The proof goes by induction, following the procedure (3) for $m$ implies (2) for $m+1$ implies (3) for $m+1$. 
For $m=1$ the assertion is guaranteed by Lemma 4 .

Suppose now that (2), (3) hold for an $m \geq 2$. Applying the identity (1) with $B(x)=x^{2}+a-m^{2}$ we have

$$
\begin{aligned}
\left(x^{2}+x\right. & \left.+a-m^{2}-m\right)\left(x^{2}+x+a-m^{2}+m\right)=(B(x)+x-m)(B(x)+x+m) \\
& \sim B(B(x)+x)=B(x) B(x+1) \sim(x-m)(x+m)(x+1-m)(x+1+m) .
\end{aligned}
$$

This together with the induction hypotheses (2) for $m$ shows that

$$
x^{2}+x+a-m^{2}-m \sim(x-m)(x+1+m),
$$

which is (2) for $m+1$.

To continue, apply identity (1) with $B(x)$ the polynomial on the left side of this relation. Then

$$
\begin{gathered}
(x-m)(x+1+m)(x+1-m)(x+2+m) \sim B(x) B(x+1) \\
=B(B(x)+x) \sim(B(x)+x-m)(B(x)+x+1+m) \\
=\left((x+1)^{2}+a-(m+1)^{2}\right)\left((x+1)^{2}+a-m^{2}\right),
\end{gathered}
$$

and (3) for $m+1$ follows if we replace $x$ by $x-1$ and apply (3) for $m$.

The proof of Lemma 5 is complete.

LEMMA 6. Let the situation of Lemma 5 be in force. Then there are integers $b_{j}, j=1, \ldots, 4$, so that $\left(x-b_{1}\right)\left(x-b_{3}\right) /\left(x-b_{2}\right)\left(x-b_{4}\right) \sim 1$, where the rational function is not identically 1 .

Proof. We may clearly assume that -1 is not a square. Suppose first that $a$ is odd or divisible by 4 . We can write it in the form $r^{2}-s^{2}$ using $r=(a+1) / 2$, $s=(a-1) / 2$; or $r=(a+4) / 4, s=(a-4) / 4$ respectively. From relation (3) of Lemma 5 with $m=|r| \geq 1$ we obtain

$$
(x-|s|)(x+|s|) /(|x-| r \mid)(x+|r|) \sim 1
$$

since then $x^{2}+a-m^{2}=x^{2}-s^{2}$ is reducible.

If $a$ is even but only divisible by 2 , it can be expressed in the form $a=$ $(c-k)(c+k-1)$. One such representation is given by

$$
a=a_{1} a_{2}, \quad c=\left(a_{1}+a_{2}+1\right) / 2, \quad k=\left(a_{2}-a_{1}+1\right) / 2
$$

provided that $a_{1}, a_{2}$ have different parity. Then $4 a=(2 c-1)^{2}-(2 k-1)^{2}$ and the quadratic polynomial $x^{2}+x+a-c^{2}+c$ is reducible since its discriminant is $(2 k-1)^{2}$. With $a_{1}=|a|, a_{2}=\operatorname{Sign} a$ we obtain from Lemma $5(2)$

$$
(x+k)(x-k+1) /(x+c)(x-c+1) \sim 1 .
$$

This completes the proof of Lemma 6.

6. In this section I show that for squarefree $g$ one can simplify the relation in Lemma 5 by acting upon a distinguished subgroup of $H(w(x))$ with a suitable ring of operators. The procedure is somewhat general.

Let $r$ be a positive integer, and let $M$ be the subgroup of $Q(x)^{*}$ generated by the first $r$ integers and the polynomials $w(P)$, where $P$ belongs to $Z[x]$ and has positive leading coefficient. Thus $M$ is possibly a little large than $\Delta(w(x))$. Let $H_{1}$ be the quotient group $Q(x)^{*} / M$, and let $\tau$ be the canonical homomorphism $Q(x)^{*} \rightarrow H_{1}$. 
Let $Y$ be the subgroup of $Q(x)$ generated by the positive integers and the rational functions of the form

$$
\psi(x)=\prod_{i=1}^{k}\left(x+c_{i}\right)^{d_{i}}
$$

with integers $c_{i}, d_{i}$. Note that for any integer $l$, the operation $\psi(x) \mapsto \psi(x+l)$ takes $Y$ into itself. In this section $\tau(Y)$ will be written additively.

We introduce a shift operator $E$ to act on $\tau(Y)$ by $E^{t} \tau(x+b)=\tau(x+b+t)$, and by linearity extend the definition so that the polynomial ring $F_{g}[E]$ acts upon $\tau(Y)$, where $F_{g}$ is the residue class ring $Z / g Z$. The $g$-torsion derived in Lemma 3 ensures that this action is well defined.

If, in the notation of Lemma $6, b=\max \left|b_{i}\right|, 1 \leq i \leq 4$, then we have

$$
\sum_{i=1}^{4}(-1)^{i+1} E^{b-b_{i}} \tau(x)=0 .
$$

LEMMA 7. Assume that $g$ is squarefree. If $r$ is fixed at a large enough value, then there is an integer $t$ so that $(E-1)^{t} \tau(x)=0$.

- Proof. To begin with assume that $g$ is a prime, so that $F=F_{g}$ is a field. Those operators in $F(E)$ which annihilate $\tau(x)$ form an ideal, nontrivial because it contains the polynomial at (4). Since $F$ is a field, this ideal is principal, generated by $\phi(E)$ say. We factorize this generator in a suitable algebraic extension of $F$,

$$
\phi(z)=\prod_{i=1}^{s}\left(z-\omega_{i}\right)^{r_{i}}
$$

with distinct roots $\omega_{i}$.

For each positive integer $d$ define

$$
\phi_{d}(z)=\prod_{i=1}^{s}\left(z-\omega_{i}^{d}\right)^{r_{i}}
$$

Since the coefficients of this polynomial are symmetric functions of the $\omega_{i}$, they are functions of the coefficients of $\phi(z)$. Thus $\phi_{d}(z)$ belong to $F[z]$. Moreover, for each value of $i$

$$
\frac{z^{d}-\omega_{i}^{d}}{z-\omega_{i}}=z^{d-1}+z^{d-2} \omega_{i}+\cdots+\omega_{i}^{d-1},
$$

so that $\phi_{d}\left(z^{d}\right) / \phi_{d}(z)$ is a polynomial with coefficients in an extension field of $F$. It is clear that these coefficients must actually belong to $F$. It follows that

$$
\phi_{d}\left(E^{d}\right) \tau(x)=0 .
$$

Let

$$
\phi_{d}(z)=\sum_{m=0}^{k} b_{m} z^{m}
$$

Then replacing $x$ in (5) by $d x$ gives (assuming that $d \leq r$ )

$$
\phi_{d}\left(E^{d}\right) \tau \mid d x=\sum_{m=0}^{k} b \tau(d x+m d)=\sum_{m=0}^{k} b_{m} \tau(x+m)
$$


since $\tau$ is a homomorphism. In other words, $\phi_{d}(E)$ also annihilates $\tau(x)$.

Since $\phi(E)$ is of minimal degree in the annihilating ideal, it must coincide with $\phi_{d}(E)$. In particular, the map $\omega \mapsto \omega^{d}$ permutes the roots of $\phi(z)$. Let

$$
\omega_{i} \mapsto \sigma \omega_{i} \mapsto \sigma^{2} \omega_{i} \mapsto \cdots \mapsto \dot{\sigma}^{h} \omega_{i}=\omega_{i}
$$

be a cycle in the permutation. Then $\omega_{i}^{d^{h}-1}=1$. We can do this for each root, and obtain an integer $\delta$ so that every $\omega_{i}^{\delta}=1$.

If $\delta \leq r$, then $\phi_{\delta}(E)$ annihilates $\tau(x)$, and $\phi_{\delta}(E)=(E-1)^{v}$ with $v=r_{1}+\cdots+r_{s}$.

Suppose now that $g$ is squarefree, with prime-divisors $p_{i}, i=1, \ldots, l$. The canonical homomorphisms $H_{1} \rightarrow H_{1} / p_{i} H_{1}$ show that $H_{1}$ is isomorphic to a direct sum of the $H_{1} / p_{i} H_{1}, i=1, \ldots, l$. For each prime $p_{i}$ we may prolong $\tau$ to the composition $\tau_{i}: Q(x)^{*} \rightarrow H_{1} \rightarrow H_{1} / p_{i} H_{1}$, and, with $F_{p_{i}}(E)$ acting on $\tau_{i}(Y)$, obtain an integer $t_{i}$ for which $(E-1)^{t_{i}} \tau_{i}(x)=0$. With $t=\max t_{i}, 1 \leq i \leq l,(E-1)^{t} \tau(x)$ projects onto zero in each $H_{1} / p_{i} H_{1}, i=1, \ldots, l$.

The assertion of the lemma is justified.

LEMMA 8. Under the conditions of Lemma $7, t=1$ may be taken.

ProOF. Let $p$ be a prime. Iterations of the map $\mu: m \mapsto(m+p-u) / p$ when $m \equiv u(\bmod p), 0 \leq u \leq p-1$, take every positive integer ultimately to 1 . Note that $p \mu(m)>m$.

Suppose that, in the notation of Lemma $7,(E-1)^{m} \tau_{i}(x)=0$. By introducing extra factors $E-1$ we reach $(E-1)^{p_{i} \mu(m)} \tau_{i}(x)=0$, from which $\left(E^{p_{i}}-1\right)^{\mu(m)} \tau_{i}(x)=$ 0 may be deduced by applying the $p_{i}$ torsion of $H_{1} / p_{i} H_{1}$. Replacing $x$ by $p_{i} x$, and arguing as in the earlier part of the proof of Lemma 7 , gives $(E-1)^{\mu(m)} \tau(x)=0$. Thus the $m$ in our hypothesis can be replaced by $\mu(m)$ and, after enough iterations of $\mu$, by 1 .

The projection of $(E-1) \tau(x)$ onto each $H_{1} / p_{i} H_{1}$ is trivial, and the proof of Lemma 8 is complete.

REMARK. In order to obtain analogues of the results of this section when $g$ is not squarefree, it would be necessary to examine the nature of the annihilating polynomial at (4) when viewed over the rings $Z / p_{i}^{\alpha_{i}} Z$, where $p_{i}^{\alpha_{i}}$ runs through the exact prime-power divisors of $g$.

7. Proof of Theorems 1 and 2 for $x\left(x^{2}+a\right)$. In this section $w(x)=x\left(x^{2}+a\right)$. From Lemma 6 there is a representation

$$
R(x)=\frac{\left(x-b_{1}\right)\left(x-b_{3}\right)}{\left(x-b_{2}\right)\left(x-b_{4}\right)}=\prod_{i=1}^{j} w\left(P_{i}\right)^{\varepsilon_{i}}
$$

where the rational function $R(x)$ is nontrivial, and the polynomials $P_{i}$ in $Z[x]$ have positive leading coefficients. Note that here $\beta=1$.

As mentioned in $\S 1$, the group $Q^{*} / \Gamma(R(n))$ is trivial for all $\theta$, and from this the triviality of $Q^{*} / \Gamma\left(n\left(n^{2}+a\right)\right)$ may now be deduced. However, we shall argue via the group $H\left(x\left(x^{2}+1\right)\right)$.

In the present circumstances $g=3$, is squarefree. From Lemma 8 we obtain a representation

$$
\frac{x-1}{x}=\lambda \prod_{i=1}^{k} w\left(F_{i}\right)^{\varepsilon_{i}}
$$


with $F_{i}=F_{i}(x)$ in $Z[x]$, and some rational number $\lambda$. Without loss of generality we may assume that no $F_{i}$ is a constant. Replacing $x$ by $x^{2}$ and forming the ratio of the two relations gives another of the form,

$$
\frac{x+1}{x}=\prod_{i=1}^{s} w\left(G_{i}\right)^{\varepsilon_{i}}
$$

with $G_{i}$ in $Z[x]$ and of positive degree.

For each integer $n$ there is a least integer $b$ so that $n+b$ is a cube. We may apply the representation (6) with $x=n, n+1, \ldots, n+b-1$ in turn, and then employ Lemma 3, to obtain both the independence of $Q^{*} / \Gamma\left(n\left(n^{2}+a\right)\right)$ of $\theta$, and its triviality.

For any polynomial $P(x)$ in $Q(x)^{*}$ relation (6) shows that with respect to the canonical map $Q(x)^{*} \rightarrow H\left(x\left(x^{2}+a\right)\right)$ we have $P(x) \sim P(x)-1$. Proceeding by induction we obtain $P(x) \sim P(x)-P(0)$, the latter being a polynomial which has a factor $x$. Thus $P(x) \sim \gamma x^{s}$ for some constant $\gamma$ and integer $s, 0 \leq s \leq 2$. From the triviality of $Q^{*} / \Gamma\left(n\left(n^{2}+a\right)\right)$ we have $\gamma \sim 1$, and $H\left(x\left(x^{2}+a\right)\right)$ is clearly cyclic of order 3 , generated by the image of $x$.

8. $G_{k}=Q^{*} / \Gamma\left(n^{k}\left(n^{2}+1\right)\right)$, and other groups. As a result of my earlier work with the groups $Q^{*} / \Gamma(F(n))$ I had postulated that such groups might satisfy the conjecture (ii) so long as $F(x)$ were not the power of another rational polynomial. (See Elliott [4, in particular Problem 12 on p. 419].) After a lecture on this subject matter which I gave at Oberwolfach, Germany, in October 1984, Lenstra (with a modification of my argument establishing the freedom of $Q^{*} / \Gamma\left(n^{2}+1\right)$ when $\theta=0$ ) and Schinzel showed that the group here denoted by $G_{2}$ contains infinitely many independent torsion elements.

Using the above result I here completely determine the groups $G_{k},|k|>1$. In this case Lemma 6 , with $\beta=k$, shows that

$$
R(x)^{k}=\left(\frac{\left(x-b_{1}\right)\left(x-b_{3}\right)^{k}}{\left(x-b_{2}\right)\left(x-b_{4}\right)}\right)^{k}
$$

has a product representation by the $w\left(P_{i}\right)$ with $w(z)=z^{k}\left(z^{2}+a\right), P_{i}$ in $Q(x)^{*}$. From the triviality of $Q^{*} / \Gamma(R(n))$ we see that with respect to the canonical map $Q^{*} \rightarrow G_{k}$ we have $m^{k} \sim 1$ for every positive integer $m$. Since trivially $m^{k}\left(m^{2}+1\right) \sim 1$, we also have $m^{2}+1 \sim 1$ for all $m$.

The primes 2 and $p, p \equiv 1(\bmod 4)$ have product representations by the $m^{2}+1$, and are thus equivalent to 1 . The primes $q, q \equiv 3(\bmod 4)$ satisfy $q^{k} \sim 1$. Suppose now that a selection of them satisfy

$$
\prod_{i=1}^{s} q_{i}^{\alpha_{i}}=\prod_{j=1}^{r}\left(n_{j}^{k}\left(n_{j}^{2}+1\right)\right)^{\varepsilon_{j}}
$$

for positive integers $n_{j}$, and integers $\alpha_{i}, 0 \leq \alpha_{i} \leq k-1$. Here each $q_{i}$ must divide a factor $n_{j}^{k}$ on the right side, and so $\alpha_{i}$ must be a multiple of $k$. This forces $\alpha_{i}=0$.

It is clear that $G_{k}$ is a direct sum of cyclic groups of order $k$, one generated by the image of each prime $q, q \equiv 3(\bmod 4)$. An elaboration of the argument given for $Q^{*} / \Gamma\left(n^{2}+b n+c\right)$ shows that the groups $G_{k}$ are independent of $\theta$.

Similar arguments show that all the groups $Q^{*} / \Gamma\left(x\left(x^{2}+a\right)^{l}\right)$ are trivial. 
Perhaps analogues of conjectures (i) and (ii) hold when $F(x)$ is replaced by (say) an absolutely irreducible polynomial in several variables, and $\Gamma$ is the subgroup generated by its values at suitably restricted points with integer coordinates.

9. Some applications. Suppose that $\chi$ is a noncubic, nonprincipal Dirichlet character, defined to some prime modulus $p$, which satisfies $\chi\left(n\left(b n^{2}+a\right)\right)=0$ or 1 for $n \leq M<p$. Applying Lemma 3 with $k=l=1$ we see that the nonprincipal character $\chi^{3}$ has value 1 on the positive integers not exceeding $c_{2} M^{1 / 3}$ for some positive constant $c_{2}$ which depends at most upon $a, b$. A device of Vinogradov (see Burgess $[\mathbf{1}]$ ) in combination with the character sum estimate

$$
\left|\sum_{m \leq H} \chi(m)\right| \leq d_{r} H^{1-1 /(r+1)} p^{1 / 4 r} \log p, \quad r=1,2, \ldots,
$$

of Burgess [2] now shows that $c_{2} M^{1 / 3}>p^{\tau}$ with a fixed $\tau>(4 \sqrt{e})^{-1}$ cannot hold for all large primes $p$.

If $\lambda>3(4 \sqrt{e})^{-1}$ and the constant $c$ is chosen suitably depending only upon $a, b$ and $\lambda$, then the interval $\left[1, c p^{\lambda}\right]$ contains an integer $n$ for which $\chi\left(n\left(b n^{2}+a\right)\right) \neq 0,1$. Since $3(4 \sqrt{e})^{-1}=.456 \ldots$ this restriction on $\lambda$ improves upon the condition $\lambda>1 / 2$ which may be deduced from a straightforward application of Weil's estimate for

$$
\sum_{n=0}^{p-1} \chi\left(n\left(b n^{2}+a\right)\right) \exp \left(2 \pi i n k p^{-1}\right)
$$

This improves upon a result of Burgess $[\mathbf{3}]$ except when $\chi$ is a cubic character or $w(x)$ has the particular form $x\left(x^{2}-s(s+1)\right)$ for some integer $s$.

Let $w(x)=x\left(x^{2}+a\right), a \neq 0$. It follows from (6) in $\S 7$ that for every pair of positive integers $m, k$, there is a representation of the form

$$
m=\prod_{i} w\left(n_{i}\right)^{\varepsilon_{i}}, \quad \varepsilon_{i}= \pm 1
$$

with $k<n_{i} \leq \mathrm{cm}^{d}$, for certain constants $d$ (depending upon $a$ ) and $c$ (depending upon $a$ and $k$ ). Suppose now that $\psi(p)$ for a prime $p$ denotes the least positive integer $n$ for which a fixed nonprincipal character $\chi$ (cubic or not) satisfies $\chi\left(n\left(n^{2}+a\right)\right) \neq 0,1$. Then this product representation together with Theorem 3 of my paper [7] show that as $y \rightarrow \infty$

$$
\frac{\log y}{y} \sum_{p \leq y} \psi(p) \rightarrow \mu
$$

for some constant $\mu$. By a fixed character $(\bmod p)$ is meant a Dirichlet character which is defined in terms of power-residue symbols (cf. Elliott [7]). An example is the Legendre symbol $\frac{n}{p}$.

Similar results may be obtained involving the rational function $x^{-1}\left(b x^{2}+a\right)$. Thus if $\lambda>3(4 \sqrt{3})^{-1}$ and the constant $c_{3}$ is chosen suitably, every interval $\left[1, c_{3} p^{\lambda}\right]$ contains an integer $n$ at which a nonprincipal character $\chi(\bmod p)$ satisfies $\chi(b n+a \bar{n}) \neq 0,1$, where $n \bar{n} \equiv 1(\bmod p)$. 


\section{REFERENCES}

1. D. A. Burgess, The distribution of quadratic residues and non-residues, Mathematika 4 (1957), 106-112.

2. __ On character sums and primitive roots, Proc. London Math. Soc. 12 (1962), 179192.

3. _ Dirichlet characters and polynomials, Proc. Internat. Conf. in Number Theory (Moscow, 14-18 Sept. 1971), Trudy Mat. Inst. Steklov 132 (1973), 203-205.

4. P. D. T. A. Elliott, Arithmetic functions and integer products, Grundlehren Math. Wiss., Springer-Verlag, New York and Berlin, 1984.

5. ___ The value distribution of reducible cubics, Canad. Math. Bull. 28 (1986), 328-336.

6. __ On representing integers as products of integers of a prescribed type, J. Austral. Math. Soc. (Ser. A) 35 (1983), 143-161.

7. On the mean value of $f(p)$, Proc. London Math. Soc. 21 (1970), 28-96.

8. R. C. Vaughan, The Hardy-Littlewood method, Cambridge Univ. Press, London, 1981.

Department of Mathematics, University of Colorado, Boulder, Colorado 80309 